\title{
Robotic esophagectomy: the Moffitt Cancer Center experience
}

\author{
Marisa Amaral, Jose Pimiento, Jacques P. Fontaine \\ Department of Surgery, University of South Florida, Tampa, FL, USA \\ Correspondence to: Jacques P. Fontaine. Department of Surgery, University of South Florida, Tampa, FL, USA. Email: jacques.fontaine@moffitt.org.
}

Submitted Dec 07, 2016. Accepted for publication Dec 21, 2016.

doi: $10.21037 /$ acs.2017.03.21

View this article at: http://dx.doi.org/10.21037/acs.2017.03.21

\section{Introduction}

The treatment of esophageal cancer is complex and labor intensive. It requires a multi-disciplinary approach and a cohesive team. Centralization of care and creation of centers of excellence with high esophageal surgical volume leads to improved outcomes $(1,2)$. Our multi-disciplinary team at Moffitt Cancer Center reached a consensus in creating a treatment algorithm for esophageal cancer in order to standardize our institutional approach and treatment.

\section{Clinical vignette}

Patients are initially staged with an upper endoscopy and computed tomography (CT) chest/abdomen/pelvis with oral and intravenous contrast. If no metastatic disease is detected on the CT scan, a positive emission tomography (PET) scan is then performed to detect lymphatic or distant metastatic disease. An esophageal endoscopic ultrasound (EUS) is also performed not only to stage the depth of invasion and regional lymphatic involvement, but also to place fiducial markers above and below the tumor to optimize planning for possible neoadjuvant radiation therapy. Of note, we treat patients with gastroesophageal (GE) junction tumors with more than $2 \mathrm{~cm}$ invasion into the gastric cardia (i.e., Siewert III tumors) as a gastric cancer.

Patients found to have T1aNo lesions undergo endomucosal resection (EMR) as their only treatment modality as long as deep and radial margins are negative. EMR is performed even for lesions clinically staged as T1bN0 as a significant percentage of them (20\%) turn out to be pathologically T1a lesions without submucosal invasion and negative margins may be obtained with EMR (3). We therefore use EMR not only as a therapeutic tool for T1aN0 lesions but also as a diagnostic tool for presumed T1bN0 lesions. Esophagectomy is reserved for patients with positive margins on EMR.

It is our institutional consensus to offer upfront esophagectomy without neoadjuvant therapy for patients with clinical T2N0M0 lesions, based on EUS and PET scan. In our series, the accuracy of staging with the combination of both PET scan and EUS for T2N0 lesions was $81.8 \%$ (3).

There is robust evidence in the literature as to the survival advantage of using neoadjuvant chemo-radiation followed by surgical resection for patients with T3N0M0 lesions or with regional lymph node involvement $(\mathrm{TxN}+\mathrm{M} 0)$ (4). Although carboplatin/pemetrexed and 45 Gy of radiation was used in the landmark CROSS trial, we routinely use cisplatin with $5 \mathrm{FU}$ concurrently with intensity modulated radiotherapy (IMRT) to 50.4 Gy with dose painting to $54 \mathrm{~Gy}$ in the esophageal bed. With this neoadjuvant regimen, we have achieved a rate of pathological complete response (pathCR) of $40 \%$ in our series (5). Surgical patients found to have a pathCR have a significantly longer median survival as compared to patients with residual disease in their surgical specimen (92.2 vs. 38.0 months) (5). Patients with GE junction tumors with upper mediastinal or supraclavicular lymph node involvement are not considered to have regional lymph node involvement and we do not offer them surgery.

We routinely repeat a PET scan 6 weeks after completion of neoadjuvant therapy to exclude metastatic disease. Unfortunately there is no sufficiently accurate modality to determine which patients achieved a pathCR with neoadjuvant therapy and could avoid surgical resection. The pooled sensitivity of PET scan for the detection of regional metastasis is only $51 \%$ (6). Therefore, even if there is no residual FDG avidity on repeat PET scan, we offer surgical resection to all patients after neoadjuvant therapy as long as they are both physically and mentally fit for surgery. 
We do not feel there is a role for a re-staging EUS after neoadjuvant therapy as tissue planes are distorted and it is not possible to differentiate scarring from active residual disease.

\section{Surgical technique}

At Moffitt Cancer Center, we prefer an Ivor Lewis esophagectomy using a robotic approach, with a right intrathoracic anastomosis. A minimally-invasive approach, as illustrated in our video, has been proven to be associated with a shorter recovery and less morbidity as compared to an open approach (7). Furthermore, the intrathoracic anastomosis avoids creating an anastomosis at the left neck, which has been found to be associated with a higher rate of dysphagia, recurrent laryngeal nerve injury and anastomotic leak (8).

For the initial abdominal phase of the Ivor Lewis esophagectomy, the patient is placed in the supine position. The gastric conduit is mobilized in the standard fashion, pedicled on the right gastroepiploic artery. A large piece of omentum is mobilized en-bloc with the conduit, and will be utilized during the thoracic phase as a vascularized flap around the conduit and anastomosis. In order to obtain the best circumferential margin around GE junction and distal esophageal tumors, the pericardium and aorta are skeletonized. A $5 \mathrm{~cm}$ wide gastric conduit is created in the abdomen but the tip of it remains attached to the esophagus in order to pull it up during the thoracic portion. Commonly, a Kocher maneuver is performed to maximize mobilization of the gastric conduit into the chest, thus allowing us to exclude more of the proximal portion of the gastric conduit, which tends to be the most ischemic portion. We inject the pylorus with 100 units of Botox for all patients and reserve a pyloromyotomy for patients with diabetes or other risk factors for gastroparesis. The left crus is kept intact; however, the right crus is completely divided to allow better angulation and drainage of the gastric conduit. A 14Fr jejunostomy feeding tube with a balloon is used for all patients. If the left pleural cavity has been violated, a 19Fr Blake drain is placed into it and brought out through a counter-stab wound in the abdominal wall. In our experience, this causes less discomfort than a traditional chest tube brought out between the ribs.

During the thoracic phase of the procedure, in order to better visualize the esophagus, the patient is placed in lateral decubitus position with an anterior tilt. Additional techniques to improve visualization include placing a retraction suture in the diaphragm, and $\mathrm{CO}_{2}$ insufflation to a pressure of $8 \mathrm{mmHg}$. The inferior pulmonary ligament is first divided to expose the inferior pulmonary vein and pericardium. In order to obtain the best circumferential margin, the dissection is not carried out along the esophagus but rather along the surrounding landmark structures. Therefore, the azygous vein, aorta, pericardium and airway are skeletonized using the robotic spatula tip electrocautery and the robotic energy device (i.e., vessel sealer). Special attention is given to avoid thermal injury to the membranous portion of the airway. The peri-esophageal and subcarinal lymph nodes are resected on bloc with the tumor. The esophageal dissection is carried up to the level of the azygous vein for GE junction tumors without long segment Barrett's esophagus. Otherwise it may be carried to the level of the thoracic inlet. If possible, all areas of Barrett's esophagus are resected. Once the esophagus is transected, the specimen is exteriorized through one of the thoracic port sites used as a $4 \mathrm{~cm}$ accessory port. A wound protector is placed in this accessory port site to avoid oncologic or bacterial contamination.

It is important to confirm correct orientation of the gastric conduit. The greater curvature with omentum should be lying along the patient's spine and the staple line of the newly formed gastric conduit should be facing the hilum of the right lung. When ready to perform the anastomosis, a $25 \mathrm{~mm}$ Orvil anvil is placed into the mouth of the patient by the anesthesia team, advanced antegrade and brought out through the staple line of the transected thoracic esophagus. The 25-mm EEA stapler with $3.5 \mathrm{~mm}$ staples is placed through a gastrotomy created in the tip of the exteriorized gastric conduit. The circular stapled endto-side esophagogastric anastomosis is created by coupling the EEA stapler and Orvil anvil by the assistant at the bedside. Both circular tissue "donuts" are confirmed to be complete, intact circles. The gastrotomy site is excluded by stapling off the tip of the gastric conduit, which, again, tends to be the most ischemic portion of the conduit. We find that this robotic-assisted approach to the anastomosis allows us to mobilize the esophagus, if necessary, all the way up at the level of the thoracic inlet, which lies very close to where a cervical anastomosis would lie. So, in our opinion, a cervical approach is rarely necessary. To mitigate pleural contamination associated with a leak from an intrathoracic anastomosis, the omentum, which was harvested en-bloc with the stomach and pedicled off the greater curvature of the gastric conduit, is brought under the gastric conduit to fill the posterior mediastinal and subcarinal spaces. It is also 
wrapped completely around the anastomosis and tacked in place with sutures. Should a leak occur, it is well contained by this omentoplasty. In addition, when dealing with GE junction tumors, we keep the azygous vein intact and create the anastomosis underneath it. This further isolates a possible leak from the pleural cavity. A 19Fr Blake drain is used to drain the pleural cavity. A $28 \mathrm{Fr}$ chest tube is only placed in addition if we suspect a post-operative air leak from the lung parenchyma.

Post-operatively, we promote an aggressive regimen of early ambulation and fluid resuscitation to maintain adequate urine output and adequate gastric conduit perfusion. We begin to trickle enteral feeding though the jejunostomy tube very early but we are quite conservative with oral intake. We perform a barium swallow on all our patients on post-operative day (POD) 8 unless there is a clinical suspicion for a leak earlier during the post-operative course. If there is no leak and adequate emptying of the gastric conduit, patients are discharged home the next day with only sips of liquids as oral intake and obtaining their caloric requirements through tube feeds cycled for 16 hours daily.

\section{Comments}

The use of the robotic platform to perform minimally invasive surgery has multiple advantages over laparoscopy and thoracoscopy. Among the advantages are the small and more dexterous endo-wristed instruments and magnified $3-\mathrm{D}$ vision. Although robotic technology is more expensive, we are convinced that like other new technology, the price will decrease over time. More importantly, we believe that it is the future of surgery. Instruments will miniaturize and more application software will be created for the computer interface.

In our institutional series of 237 consecutive roboticassisted esophagectomy procedures, there is a leak rate of $15 \%$. The majority of anastomotic leaks were clinically silent, only found on barium swallow and required no intervention. Most of these patients went home, remained NPO, with plans to return a week later for a repeat barium swallow. Only $4 \%$ of patients had a leak which required additional drainage, stent placement or re-operation. The majority of anastomotic leaks occur between POD 4 to 7 . Therefore, a barium swallow performed on POD 5 would miss anastomotic leaks that developed later on POD 8. Although there are financial and administrative pressures and perhaps egotistical reasons to discharge patients home early after a minimally invasive esophagectomy, we prefer to keep patients until the anastomosis has been reliably studied, and until patients and their families are comfortable with enteral feedings and physical therapy. A significant portion of our patients live at quite a distance from the Center and, and should post-operative problems occur early after their discharge home, re-evaluation and readmission to the Cancer Center can be logistically very challenging. Lowering readmission rates to our Center or to outside hospitals in the community is part of our rationale for routinely keeping patients in-house until POD 9.

The technically successful robotic esophagectomy is only a component of building a successful esophageal cancer treatment program. An experienced, innovative and cohesive multidisciplinary team with appropriate financial and administrative support is essential.

\section{Acknowledgements}

None.

\section{Footnote}

Conflicts of Interest: The authors have no conflicts of interest to declare.

\section{References}

1. Casson AG, van Lanschot JJ. Improving outcomes after esophagectomy: the impact of operative volume. J Surg Oncol 2005;92:262-6.

2. Finks JF, Osborne NH, Birkmeyer JD. Trends in hospital volume and operative mortality for high-risk surgery. $\mathrm{N}$ Engl J Med 2011;364:2128-37.

3. Luu C, Garcia-Henriquez N, Klapman J, et al. Accuracy of clinical staging with EUS for early stage esophageal cancer: Are we denying patients beneficial neoadjuvant chemo-radiation? J Clin Oncol 2016;34:abstr 163.

4. van Hagen $P$, Hulshof MC, van Lanschot JJ, et al. Preoperative chemoradiotherapy for esophageal or junctional cancer. N Engl J Med 2012;366:2074-84.

5. Blackham AU, Yue B, Almhanna K, et al. The prognostic value of residual nodal disease following neoadjuvant chemoradiation for esophageal cancer in patients with complete primary tumor response. J Surg Oncol 2015;112:597-602.

6. van Westreenen HL, Westerterp M, Bossuyt PM, et al. Systematic review of the staging performance of 
18F-fluorodeoxyglucose positron emission tomography in esophageal cancer. J Clin Oncol 2004;22:3805-12.

7. Nguyen NT, Follette DM, Wolfe BM, et al.

Comparison of minimally invasive esophagectomy with

Cite this article as: Amaral M, Pimiento J, Fontaine JP. Robotic esophagectomy: the Moffitt Cancer Center experience. Ann Cardiothorac Surg 2017;6(2):186-189. doi: 10.21037/ acs.2017.03.21 transthoracic and transhiatal esophagectomy. Arch Surg 2000;135:920-5.

8. Levy RM, Wizorek J, Shende M, et al. Laparoscopic and thoracoscopic esophagectomy. Adv Surg 2010;44:101-16. 